\title{
Du kitsch à la BNU : les objets du fonds Abraham Moles
}

De quelques idées, réflexions et sensibilités molésiennes qui ont présidé à l'invention de l'exposition Kitsch, art du bonheur

\section{Thomas Soriano}

\section{(2) OpenEdition} Journals

Édition électronique

URL : http://journals.openedition.org/rbnu/602

DOI : 10.4000/rbnu.602

ISSN : 2679-6104

Éditeur

Bibliothèque nationale et universitaire de Strasbourg

Édition imprimée

Date de publication : 1 novembre 2018

Pagination : 44-51

ISBN : 9782859230784

ISSN : 2109-2761

Référence électronique

Thomas Soriano, "Du kitsch à la BNU : les objets du fonds Abraham Moles », La Revue de la BNU [En ligne], 18 | 2018, mis en ligne le 01 juillet 2019, consulté le 11 décembre 2020. URL : http:// journals.openedition.org/rbnu/602 ; DOI : https://doi.org/10.4000/rbnu.602

\section{(c) (i) (9)}

La Revue de la BNU est mise à disposition selon les termes de la Licence Creative Commons Attribution - Pas d'Utilisation Commerciale - Partage dans les Mêmes Conditions 4.0 International. 


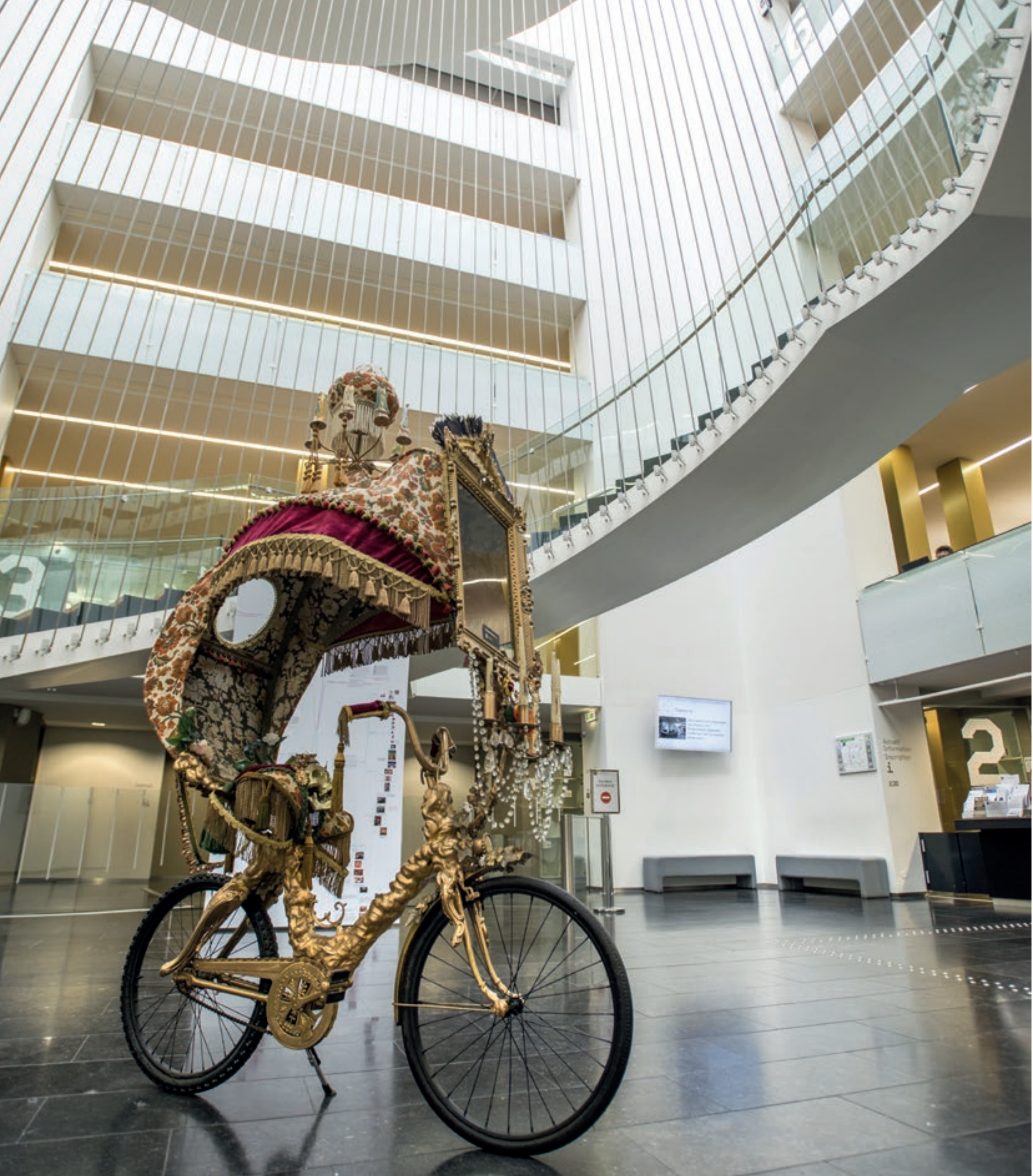




\section{DU KITSCH À LA BNU : LES OBJETS DU FONDS ABRAHAM MOLES \\ De quelques idées, réflexions et sensibilités molésiennes qui ont présidé à l'invention de l'exposition Kitsch, art du bonheur ${ }^{1}$}

PAR THOMAS SORIANO

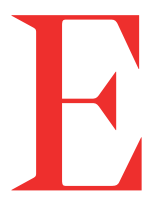

n septembre 2017 s'est tenu à l'Université de Strasbourg le colloque intitulé Abraham Moles et l'École de Strasbourg ${ }^{2}$. Il a été accompagné d'une exposition-hommage à la BNU, intitulée Kitsch, art du bonheur et qui a été montrée en septembre-octobre $2017^{3}$. Nous devons à Elisabeth Rohmer-Moles la belle idée de cette exposition sur le thème du kitsch, phénomène social et esthétique universel, dont Moles livra la première étude en 1971 (Psychologie du kitsch, l'art du bonheur, Paris, Mame $\left.{ }^{4}\right)$.

Il s'est agi, à partir de la précieuse petite collection d'objets recueillis avec patience et passion, devine-t-on, par Moles et présente dans le fonds Moles de la $\mathrm{BNU}^{5}$, d'imaginer, de provoquer un « acte rare " celui qui condense émotion et originalité dans la trace qu'il laisse - sous la forme d'une " exposition plaisir ». Était-ce une exposition sur le kitsch, était-ce une exposition kitsch ? Forcément les deux, puisque, nous dit Moles, la relation au kitsch - et l'art qui en résulte - est profondément et naturellement pédagogique : si « le bon goût s'établit socialement contre, à travers et donc par la voie du mauvais goût ", le kitsch nous apprend alors à coup sûr les critères sémantiques de l'œuvre...

\section{— Ritualisation et surdétermination}

Un autre acte décisif, sous forme d'aubaine, fut la participation enthousiaste à ce projet de Sébastien Fritschy, jeune et brillant artiste plasticien dont toute la réflexion et la production interrogent le kitsch contemporain. Nous avons eu la chance, enfin, de rencontrer la muséographe mexicaine Mar Fuentes Lanning qui nous proposa d'illustrer et d'expliciter par l'exemple les pratiques de ritualisation, au Mexique, de certaines célébrations et festivités par l'introduction, l'adjonction et la coagulation d'objets kitsch, lesquels finissent par s'imposer jusque dans les gestes de la vie quotidienne : objets de table comme salière ou poivrière, autels religieux miniature, panoplie pour le quinzième anniversaire des jeunes filles... Ces «créations ", commanditées ou élaborées de toute pièce, mimant une « arte povera » sans préméditation, s'insinuent jusque dans les rites d'initiation, constituant ainsi le décor, le support vivant des déclarations de foi et d'amour.

Le second volet de l'exposition, celui de Sébastien Fritschy, a présenté des œuvres d'art contemporaines, pour certaines inédites, élaborées avec patience, conscience et obstination. Celles-ci comprennent intègrent sciemment - toutes les caractéristiques de l'objet kitsch pointées par Moles : aliénation et mise en condition de l'homme par les objets qui l'entourent, glissement du rapport d'usage au rapport d'amour pour l'objet, puis à celui d'une intégration nécessaire où lui- 
même se range dans un réseau complexe de relations où aucune différence n'existe plus entre l'un et l'autre.

Parodie, ironie dans ce travail artistique ?

Nous dirions plutôt "surdétermination » dans les traits et les gestes, ainsi que nous avons pu parler ailleurs ${ }^{6}$ de la manière dont certains artistes détournent avec quelque insolence des signes graphiques politiques fascisants (comme dans le cas du collectif slovène IRWIN, par exemple) : la surdétermination, mieux que l'ironie qui n'userait plus que de métaphores émoussées, ne pourrait-elle ainsi devenir la figure de rhétorique la plus efficace de la "dénonciation"?

L'exposition présentait une agrégation maniaque, obsidionale de tous les facteurs du kitsch : entassement ou facteur de frénésie, romantisme du fantastique, confort et culture mosaïque (comme a pu le montrer Moles dans Psychologie du kitsch, op. cit., p. 86). Une telle systématisation, du côté de l'auteur artiste, établit sans doute ici un léger écart avec l'idée d'un kitsch molésien vu et vécu comme un vice caché, comme " un vice tendre et doux »... Peut-être ce vice perdu se réinvestit-il subrepticement dans l'esthétique de la réception ? Et enfin, s'annonce aussi en la matière quelque effet déceptif pour l'auteur quand on sait que, selon Moles, nul ne peut être totalement kitsch en en prenant conscience!

\section{Mathématiques du kitsch}

L'exposition présentait, comme dans une (dé) monstration inchoative, quelques objets, triés sur le volet, de la magnifique collection d'A. Moles et d'autres collections particulières "savamment " assemblées - savamment signifiant simplement que nous avons cédé là à l'appel sauvage de la figure de l'abondance, de l'accumulation, de l'entassement, règle d'or de toute composition kitsch, tout en intériorisant l'idée que, pour être sans dommage, cet entassement des objets kitsch suivrait la loi de compression des gaz qui s'énonce ainsi :

$$
\mathrm{p}(\mathrm{v}-\mathrm{b})=\mathrm{cte}
$$

pression kitsch $\mathrm{x}$ (volumes - covolumes $)=$ constante,

les co-volumes désignant les objets qui se touchent (ibid., p. 57). Nous avons été fidèles en ceci à cette "pression kitsch » si sensible intuitivement, dit Moles, quand on pénètre dans certains appartements bourgeois! "Savamment assemblées " veut tout de même dire l'espoir insensé d'une source de surréalisme combinatoire inconscient !

Pour ce qui est de la typologie des formes kitsch elles-mêmes, au moins pour les formes élémentaires, celle du kitsch 1900 par exemple, leurs courbes nous viendraient du Modern style, le style nouille : les spaghettis en train de cuire suivraient des équations de déformations voisines. «Elles comportent des inflexions nombreuses et peu de décrochages ou de discontinuités, sauf en fin de motif ". Régies par des équations avec points d'inflexion à dérivée seconde nulle ( $\mathrm{D}$ au carré $\mathrm{x}$ Y / D x $2=0$ ), ces courbes, répétitives, redondantes, à les considérer comme équipotentielles d'un champ entre deux contours linéaires, permettent d'imaginer un mode de génération mécanique. La machine à faire du kitsch, nous dit Moles, est concevable : en suivant la résolution de l'équation de Laplace, utilisée en hydrodynamique (delta indice $2 \times \mathrm{V}=0$ ), en partant de contours polygonaux à empattements (ibid., p. 50)! Ces quelques exemples d'investigation à forte densité mathématique, scientifique ou simplement épistémologique, qui ont pu faire dire à Umberto Eco, commentant l'ouvrage Théorie de l'information et perception esthétique, que Moles «est un auteur parfois difficile », devraient suffire à montrer combien le kitsch (des mots allemands kitschen, «bâcler », et Kitsch, « camelote », vers 1860) s'oppose à la simplification : « le kitsch est un art puisqu'il agrémente la vie quotidienne d'une série de rites ornementaux qui la décorent et lui donnent cette exquise complication, ce jeu élaboré, témoignage des civilisations avancées » (ibid., p. 20).

\section{Le gadget et la philosophie de l'objet}

Le philosophe de l'« objectité ", comme aime à se présenter François Dagognet ${ }^{7}$, ne dédaigne pas le gadget. Que ce mot désigne à l'origine, dans l'argot de la marine, un accessoire mécanique, une «bricole " ou qu'il se rattache à "gâchette " et même à "gagée " (de gage, équivalent de salaire, vers 1870) n'exclut pas, pour le néo-kitsch, une origine différente et plus récente : M. Gaget, industriel parisien et patron de la 


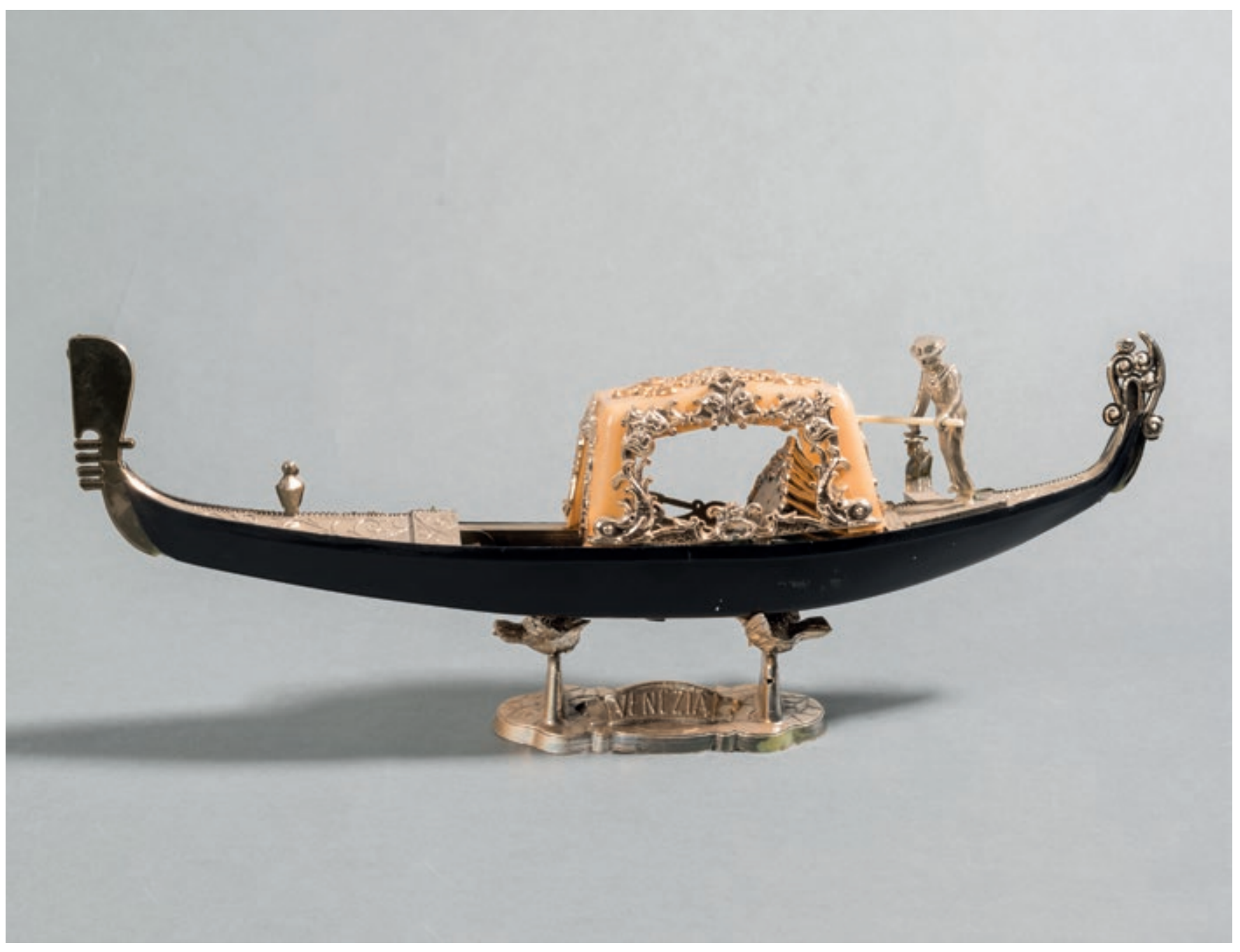

Gondole de Venise, plastique noir, doré et argenté, habitacle imitation corne (années 1960 ? ; coll. BNU) 


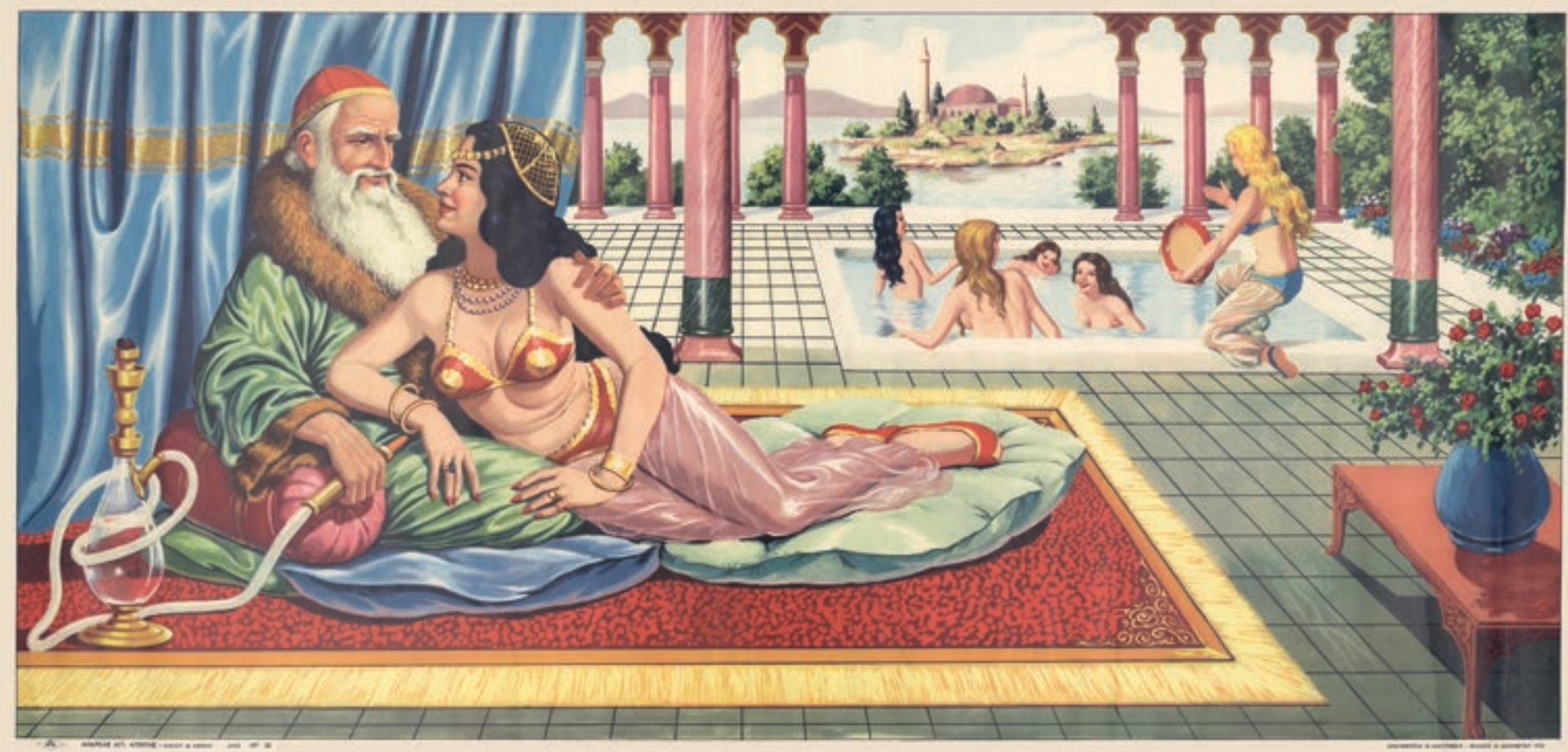

Chromolithographie orientaliste

(sans auteur ni titre ; éd. Andreas Agg. Apergis, Athènes, 15 décembre 1972 ; coll. BNU) 
société chargée de construire et de fondre la statue "La liberté éclairant le monde " de Bartholdi, aurait commercialisé des statuettes miniatures dont auraient raffolé les Américains : d'où une possible idiomatisation de Gaget en gadget...

Appartenant souvent à l'industrie des souvenirs (fanions, cendriers, briquets, porte-clés...), le gadget, par son caractère artificieux, apparaît comme une maladie de la fonctionnalité ( « un objet pour chaque fonction ») : il établit un contact kitsch entre l'univers des situations, celui des actes et celui des objets (Psychologie du kitsch, op. cit., p. 213). Qu'il nous procure le plaisir de connaître un reflet du monde technique, qu'il joue un rôle mnésique, cet « objet d'objet » fait fonction d'image. Trop souvent décrié, comme nous le rappelle F. Dagognet, il allie ludique, factice et adventice dans un climat somme toute guère différent de certaines expériences de foire ayant favorisé la découverte de phénomènes électro-magnétiques, ou de celles préfigurant l'invention de la « camera obscura ». Dans la vie courante, si nous acceptons l'idée que le gadget vient assumer une micro-fonction du quotidien, une fonction répétitive, c'est en faisant augmenter la part de jeu dans les actions les plus banales qu'il produit le kitsch, en établissant une disproportion entre les moyens et les fins : une attention portée aux moyens pour un résultat quasi ridicule. Ainsi le gadget peut prétendre à la réduction de tous les budgets! Les œuvres récentes, somptuaires et monumentales, d'un Jeff Koons, inversant en quelque sorte le schéma du gadget, en jouant plus sur la préciosité que sur une distanciation ludique, relèvent-elles encore du kitsch, comme on le dit ?

Quelques visiteurs de l'exposition Kitsch, art du bonheur nous ont fait part de leur surprise de voir figurer dans cet ensemble d'objets quelques photos, reproduites avec soin à petite échelle (avec l'aimable autorisation de l'artiste), des actions et performances sous forme d'opérations chirurgicales d'ORLAN. Elles accompagnaient en fait un beau texte de Moles sur ORLAN, "Fremd im eigenen Körper ${ }^{8}$ : étrangère en son propre corps, ORLAN, nous dit Moles, en modifiant son corps, son identité (?) par la chirurgie, en étant sujet-témoin de ses aventures, en donnant des valeurs esthétiques à une salle d'opération fonctionnelle, réinvente le rite où le prince Victime se trouverait être resté le prince Roi... Chiasme de la gravité et de la quotidienneté, distancia- tion par rapport au corps à la mesure de sa résection, de son ouverture ? Incontestablement, les différentes figures d'ORLAN présentées dans l'exposition, celles aussi d'une nonne dénudant un sein, ou d'une mariée croisant luxure et drapé - le titre des œuvres est parfois tout un voyage, ainsi «Le Drapé, le Baroque ou Sainte ORLAN libertine se joue des photos de mariage »- ne sont pas loin d'inviter à un kitsch d'une complexité... diabolique.

Le monde des objets, "plein de prodiges, de sédimentation et d'arrangements ingénieux » (F. Dagognet), s'il est une invitation constante à l'appropriation, au fétichisme même pratiqué par le collectionneur, implique une dimension ostentatoire : « l'objet est d'abord montré pour qu'on veuille le posséder, puis il est possédé pour être montré en réponse ». Le regard s'attardant sur lui est d'autant plus rafraîchissant que s'accroissent aujourd'hui contingence, instabilité et dissipation. Dans l'« ustensilité » de l'objet, qu'on aurait pu écrire aussi « utensilité " comme dans le latin « uti " ${ }^{9}$ (racine d'« outil»), le poète Francis Ponge voit l'attraction exercée par le mot voisin "ostensible " : même de pacotille, les objets sont dans nos maisons «pendus un peu comme des ex-voto ${ }^{10}{ }^{1}$... Il s'agit bien de hâter la rencontre fervente entre l'Homme et son univers, délivrer le monde des objets de toute sujétion (!), laisser les objets sortir de leur mutisme.

\section{Disproportion des dimensions, l'aigre et le doux}

Arc de triomphe sur porte-clé, éléphant en porcelaine miniature, souris gigantifiée et stylisée en bronze, la dimension porte sur la dérivée d'une grandeur géométrique. Une autre opposition dialectique est celle d'Eros / de Thanatos. D'un côté le kitsch doux-sucré, les figures de jardin, les poupées rose chair, les monuments en sucre de pâtisserie... De l'autre, le kitsch aigre, les terribles crânes mexicains en sucre, les squelettes en matière plastique américains... avec parfois un génial court-circuit, "Witz » freudien en trois dimensions, dans une figurine de nain de jardin tombé à terre, un poignard dans le dos (collection de Roland Graff)... 
Suggérons même une tentative de retournement : et si «l'entassement, la synesthésie, la médiocrité éventuellement dorée, l'angoisse possessive, la disproportion entre les moyens et les fins, le romantisme, un souvenir du rococo, une touche de maniérisme " (A. Moles), en résumé les composants du bouillon kitsch n'étaient plus vus comme symptômes d'aliénation pour des "indigènes " pris dans les rets de la société de consommation, que pas même un humour partagé ne parviendrait à soulager ? Une fois le kitsch assumé, peuvent-ils être vus comme un syndrome de résistance, faite d'ingénuité, d'effronterie et de sardonisme, simple résistance à la fonctionnalité cybernétique et à l'ascétisme destructeur?

\section{Résister : entre facteur Cheval et D'Annunzio}

Il y a du kitsch au fond de chacun de nous. Qui douterait, en voyant les "palais " construits récemment, sans plans dessinés, par la communauté rom calderash, enrichie par le commerce des métaux, dans le village roumain de Buzescu, qu'ils ne relèvent, dans leurs délires architecturaux époustouflants, d'une affirmation de soi, « d'un orgueil de l'œuvre accomplie, pour des êtres coextensifs à leurs possessions " (A. Moles) ? Celui-ci eût-il pu les faire figurer dans son iconographie du kitsch ? Avec leurs créations à mille lieux du palais idéal d'un facteur Cheval ou du Vittoriale degli Italiani d'un Gabriele d'Annunzio, les Roms, sans peine pourtant, "s'égalent eux aussi aux potentats hindous dans la mesure où ils ne sortiraient plus des limites de leur petit royaume " (id.)...

«Si le kitsch est permanent comme le péché, il y a une théologie du kitsch " (id.) - tout au moins une rédemption dans une fierté, peut-être une identité recouvrées, en lesquelles, dans une irrésistible extrapolation, nous pourrions voir comme une résistance à un pouvoir central, une langue dominante, dans la perpétuation même de coutumes chamaniques... à l'instar des peuples premiers que l'ethnologue Jean Malaurie nous fait respecter et aimer ${ }^{11}$.

Trois catégories d'objets ont donc été montrées dans l'exposition, des créations, des objets ritualisés, des objets collectionnés, en un lieu "détourné », une salle de bibliothèque, hébergeant ainsi avec bonheur des œuvres qui de paroles qu'elles furent, signes sont devenues. Un lieu n'est donc vraiment lieu que dans les interstices (de liberté, dirait encore Moles) qui s'y produisent. En effet, comme l'écrit le philosophe Henri Maldiney, « l'art n'existe qu'à entretenir la perpétuité de l'instant $"{ }^{12}$.

En ce qui concerne les objets du fonds Moles de la BNU, semblable action souligne la pertinence qu'il $\mathrm{y} \mathrm{a}$, pour une bibliothèque, à recueillir et valoriser des « documents " moins habituels que les imprimés certes, mais n'en faisant pas moins partie intégrante des fonds qu'elles hébergent : les lignes qui précèdent ont montré à quel point ces documents reflétaient - souvent comme autant de paradigmes - la pensée, les centres d'intérêt et les instruments de travail d'un chercheur. C'est aussi cela que l'exposition donnait à voir.

\section{NOTES}

1- Ce texte reprend, remanie et développe les thèmes du discours que nous avons prononcé lors de l'inauguration de l'exposition le 29 septembre 2017 à la BNU.

2- Le colloque s'est déroulé les 28, 29 et 30 septembre 2017 à l'occasion $\mathrm{du} 25^{\mathrm{e}}$ anniversaire de la disparition de Moles. Pionnier de la transdisciplinarité - qu'il opposait à ce qu'il appelait la culture mosaïque -, Abraham Moles (1920-1992) fut tour à tour et en parallèle ingénieur, acousticien, philosophe, psychologue, sociologue, théoricien et historien de l'art. Pour plus d'informations, voir le site gream.unistra.fr.

3- L'exposition s'est déroulée du 29 septembre au 20 octobre 2017 à la BNU. Elle a été réalisée avec la participation de Sébastien Fritschy, de Mar Fuentes Lanning et d'ORLAN. Pour plus d'informations, voir www.bnu.fr.

4- Psychologie du kitsch a été traduit en plusieurs langues et réédité plusieurs fois, dont une réédition récente chez Pocket (2016).

5- Le fonds Moles a été donné à la bibliothèque par sa veuve, Elisabeth Rohmer-Moles, en 2013. Comprenant toute la bibliothèque et les instruments de travail du chercheur, il a gardé de ce fait toute sa cohérence. On l'estime à environ 7000 pièces, et il contient, au-delà des documents imprimés traditionnels, bon nombre d'objets dont ceux dont il est question dans cet article. Voir à ce sujet La Revue de la $B N U, \mathrm{n}^{\circ} 10,2014$, p. 86-91.

6- Thomas Soriano, in « Seminario Arte contemporânea da Europa central e oriental », Université de Porto Alegre, Brésil, 2012 (www.ufrgs.br)

7- In Éloge de l'objet, Vrin, 1989

8- In Inter, 54, 1992

9- Verbe latin signifiant « utiliser », « se servir de »

10- Ponge, Francis, Le Grand recueil, 1961

11- Cf. Oser, résister, CNRS éditions, 2018

12- Maldiney, Henri, Art et existence, Klincksieck, 1985 


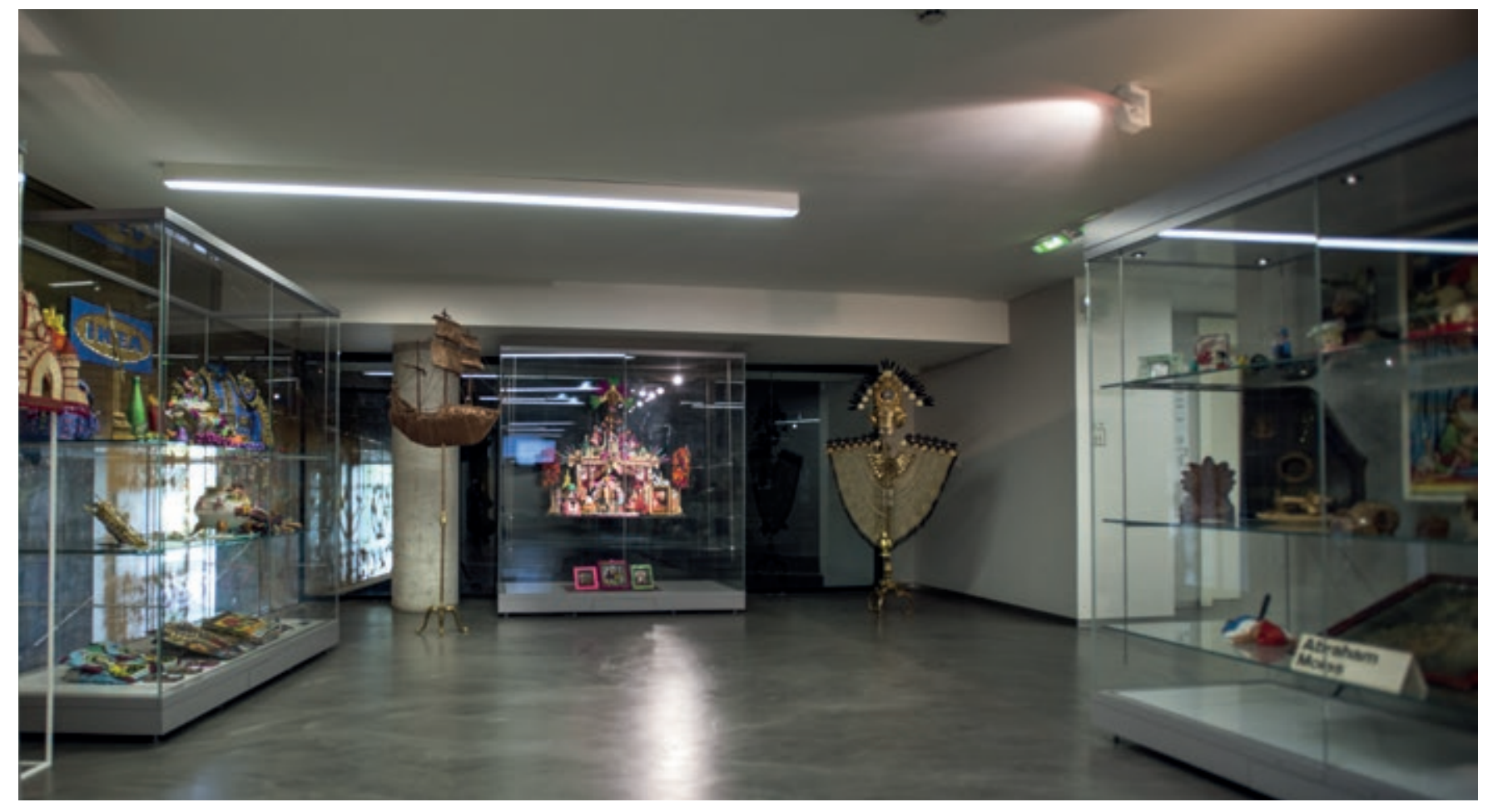

Vue d'ensemble de l'exposition avec la vitrine contenant les objets du fonds Abraham Moles

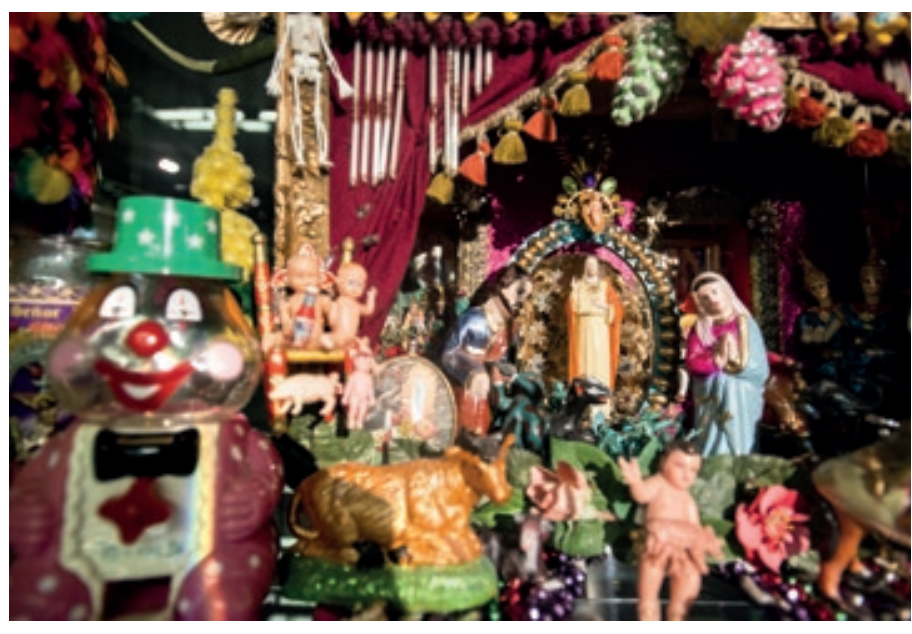

Autel religieux, œuvre de Sébastien Fritschy (2016) présentée dans l'exposition Kitsch, art du bonheur à la BNU

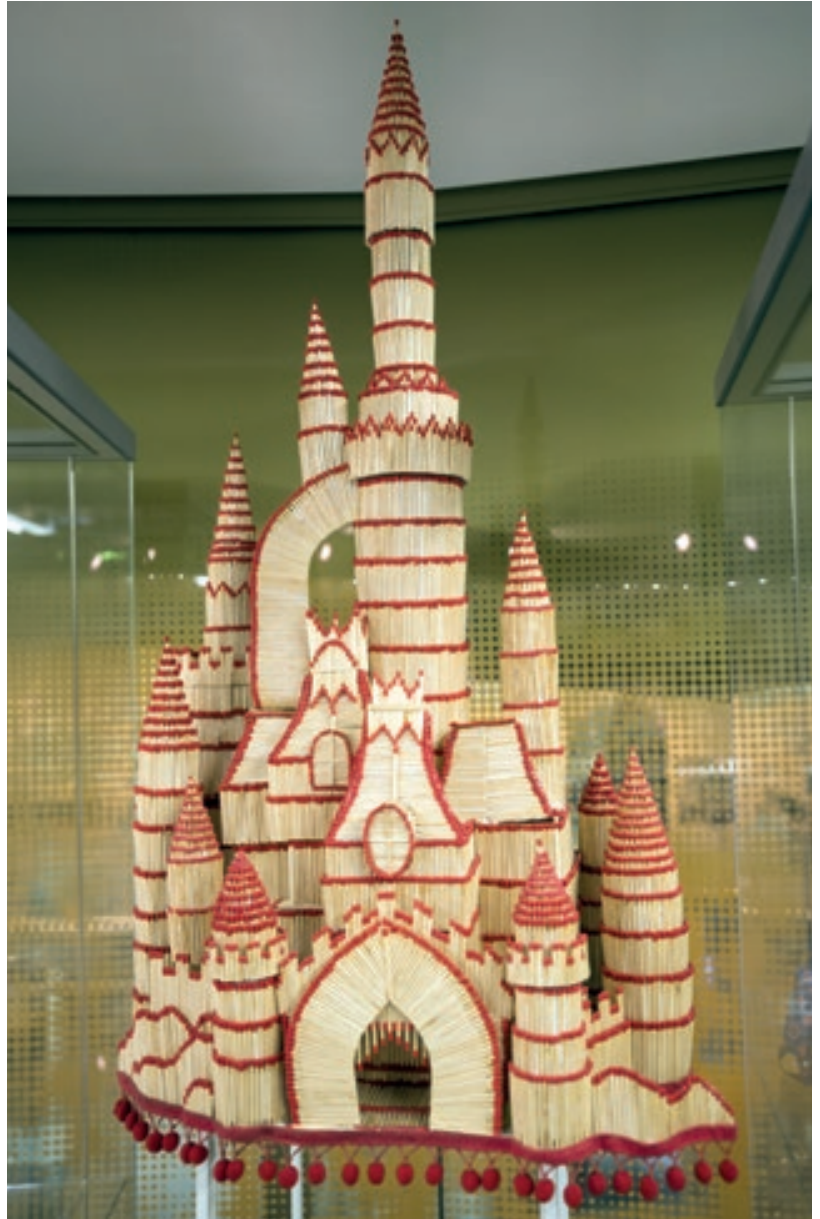

Palais en allumettes, œuvre de Sébastien Fritschy (2016) présentée dans l'exposition Kitsch, art du bonheur à la BNU 